\title{
Diastolic Echocardiographic Parameters in Predicting Outcome of Radiofrequency Catheter Ablation of Atrial Fibrillation
}

\author{
Antti Hakalahti ${ }^{*}$, Paavo Uusimaa $^{2}$, Kari Ylitalo $^{1}$ and M.J. Pekka Raatikainen ${ }^{3}$ \\ ${ }^{1}$ Institute of Clinical Medicine, Department of Internal Medicine, Division of Cardiology, University of Oulu, P.O. Box \\ 5000, FI-90014 Oulu, Finland \\ ${ }^{2}$ Department of Internal Medicine, Länsi-Pohja Central Hospital, FI-94100, Kemi, Finland \\ ${ }^{3}$ Heart Center Co., Tampere University Hospital, P.O. Box 200033521, Tampere, Finland
}

\begin{abstract}
Aims: Due to frequent relapses after initial ablation, there is need to develop accurate and practical preoperative means to predict atrial fibrillation $(\mathrm{AF})$ ablation outcome.

Methods: We evaluated the power of diastolic echocardiographic parameters in predicting the outcome of AF ablation. Forty-nine patients with symptomatic and drug-resistant paroxysmal or persistent $\mathrm{AF}$, who underwent wide circumferential pulmonary vein isolation, were included in the study. Clinical and echocardiographic (e.g., left atrial size, left ventricular size and ejection fraction and several diastolic parameters) data were collected prospectively.

Results: After single ablation procedure, 18 patients (37\%) were free of AF without antiarrhythmic medication (group 1), whereas 31patients had symptomatic or asymptomatic AF relapses or needed antiarrhythmic medication (group 2) during the 12 months follow-up period. There was no difference in age ( $52 \pm 8$ vs $54 \pm 8$ years), body mass index ( $26 \pm 4 v s 28 \pm 4$ ), comorbidity and procedural factors between the groups, but history of cardioversion was more common in group 2 (73\% vs $39 \%, P=0.032$ ). In multivariate analysis, elevated ratio of early diastolic mitral flow velocity and mitral annular early diastolic velocity (E/e' ratio) was the most powerful predictor of AF recurrence (OR 1.911; 95\% CI 1.046-3.493, $P=0.035$ ).

Conclusions: In our study, diastolic dysfunction measured by E/e' ratio was an independent predictor of AF recurrence after ablation. As a non-invasive and readily available measurement, the E/e'ratio may help to identify the patients in risk for treatment failure and to refine treatment strategy.
\end{abstract}

Keywords: Atrial fibrillation, catheter ablation, echocardiography, outcome.

\section{BACKGROUND}

Atrial fibrillation (AF) is a deleterious arrhythmia with multiple aetiological factors. It requires complex and individualized treatment. During the last decade, radiofrequency catheter ablation (RFA) has become an important treatment option in patients with drug-refractory AF [1-6]. It is well established that the efficacy of RFA is better among patients with paroxysmal compared to those with persistent or long-standing persistent AF [7, 8]. Traditionally, the decision about ablation has been made on the basis of procedural risk, patient's age, cardiac comorbidities, left atrial (LA) size and the type of AF (e.g., paroxysmal, persistent, long-standing persistent) [1]. However, relapses after the initial ablation are common also in patients with no comorbidities, and multiple procedures are required to achieve clinical efficacy in about $20-40 \%$ of the patients with lone paroxysmal AF [8-11].

*Address correspondence to this author at the Institute of Clinical Medicine, Department of Internal Medicine, Division of Cardiology, University of Oulu, P.O. Box 5000, FI-90014 Oulu, Finland; Tel: +358407777497;

E-mail: anttika@mail.student.oulu.fi
Hence, there is urgent need to identify patients with more complex arrhythmic substrate in order to find those who are most likely to benefit from ablation therapy and to guide ablation strategy. Mahnkopf, et al. recently demonstrated that the extend of fibrosis in the atria detected by a specific magnetic resonance imaging (MRI) technique correlated with the success of AF ablation [12]. As a non-invasive and more readily available diagnostic tool, echocardiography might provide a more practical preoperative means to predict AF ablation outcome. In this study, we evaluated the prognostic value of diastolic echocardiographic parameters in predicting the outcome of RFA of AF.

\section{METHODS}

\section{Study Population}

A total of 49 patients with symptomatic and drug refractory AF, who underwent wide circumferential pulmonary vein isolation (PVI) procedure, were included in this prospective study. AF was paroxysmal in 25, persistent in 17 and long-standing persistent in 7 patients, respectively. Patients with previous left-sided catheter ablation, open heart surgery, severe structural heart disease (e.g., congenital heart disease, prior myocardial infarction, chronic heart failure, 
severe valvular heart disease), significant extra-cardiac comorbidities, enlarged left atria $(>50 \mathrm{~mm})$ or intracardiac thrombi in transesophageal echocardiography were excluded. The study was approved by the local ethical committee and a written consent for participation was obtained from all patients.

\section{Echocardiography}

Transthoragic and transesophageal echocardiography was performed within 24 hours before the ablation procedure by an experienced cardiologist. Echocardiography was performed according to the guidelines of American Society of Echocardiography. A Philips Sonos $7500^{\circledR}$ ultrasound system (Philips Medical Systems, Eindhoven, The Netherlands) was used for all examinations. Left atrial and left ventricular (LV) dimension were measured by M-mode in parasternal view and left ventricular ejection fraction (LVEF) by the Simpson's method. Valvular structure and function was evaluated with two-dimensional and Doppler ultrasound modalities. Transmitral flow and propagation velocities were acquired as in normal clinical practice. Pulmonary vein (PV) flow measurements were performed by placing the sample area of pulsed-wave Doppler in the right upper PV. Color tissue Doppler (TD) cine loops (at least 3 cycles) were obtained from apical four-chamber view at 100 $\mathrm{mm} / \mathrm{s}$ sweep speed and analysed later for basal septum TD velocities with a specific program (QLAB, Philips Medical systems).

\section{Medication}

Oral anticoagulation with stable international normalized ratio of 2.0 or higher was ensured at least three weeks before the ablation. After transseptal puncture, intravenous heparin was administered according to our institutional standards without monitoring the activated clotting time [13]. Antiarrhythmic drugs (AAD) were not discontinued at the time of the echocardiographic examination and the ablation procedure. After the ablation, antiarrhythmic medication was allowed upon physician's discretion during the initial three months blanking period. Thereafter, supplementary AAD therapy was discouraged. Angiotensin converting enzyme inhibitors and angiotensin II receptor blockers were used commonly in hypertensive patients.

\section{Ablation Procedure}

A three-dimensional electroanatomical map of the left atrium and the PVs was constructed using a nonfluoroscopic navigation system $\left(\right.$ Carto $^{\mathrm{TM}}$, Biosense Webster, Diamond Bar, CA, USA) as described previously [13]. The ostia of the PVs were identified by fluoroscopic visualisation with the help of PV angiography or previously acquired computed tomography or MRI image. PVs were isolated pairwisely by creating a wide circumferential line around the right- and left-sided veins using an open-irrigated ablation catheter (Navistar Thermocool, Biosense Webster). Electrical isolation of the PVs was confirmed by careful remap of the lines and PV ostia or by using a circumferential diagnostic catheter.

\section{Follow Up}

Clinical controls including symptom enquiry, evaluation of medications and 24 hour Holter monitoring were performed at three, six and twelve months. Patients were also informed to contact the hospital in case of arrhythmia symptoms for Holter monitoring and clinical evaluation. Early AF relapses during the three-month blanking period were treated by electrical cardioversion and antiarrhythmic drug therapy if necessary. In patients free of AF, AADs were discontinued three months after the procedure. Treatment success was defined as freedom of symptoms and documented AF or atrial tachyarrhythmia without concomitant AAD therapy after the blanking period.

\section{Statistical Analysis}

IBM SPSS Statistics 20.0 (IBM Corp., Armonk, NY, USA) was used for statistical analysis. Nominal variables are expressed as numbers or percentages and compared by Fisher's exact test or Chi-square test when appropriate. Ordinal variables are presented as mean \pm SD and compared with Wilcoxon Rank sum test. Continuous variables are expressed as mean \pm SD and compared with independent variables $t$ test. Variables with a $P$ value of $<0.1$ in univariate analysis were further analysed in multivariate model (logistic regression). All tests were two-sided and a $P$ value of $<0.05$ was considered statistically significant.

\section{RESULTS}

At one year after a single AF ablation procedure 18 out of 49 patients $(37 \%)$ were free of AF without AADs (group $1)$, whereas 31 patients $(63 \%)$ had recurrent symptoms, documented AF relapse or need for AADs after the three month blanking period (group 2). There were no procedurerelated complications. The baseline characteristics of the groups were similar in age $(52 \pm 8$ vs $54 \pm 9, P=0.47)$, comorbidities, previous antiarrhythmic medication $(1.5 \pm 0.8$ vs $1.6 \pm 1.1, P=0.82)$ and antihypertensive drugs $(1.1 \pm 0.8$ vs $1.1 \pm 0.7, P=0.93)$. History of cardioversion $(\mathrm{CV})$ was less common in Group $1(39 \%$ vs $73 \%, P=0.032)$ and all seven female patients had AF recurrence (Table 1). The majority of the patients had no underlying diseases $(67 \%)$. Hypertension was present in 10 patients $(20 \%)$, coronary artery disease in 3 patients $(6 \%)$ and lung disease in 3 patients $(6 \%)$. About half of the patients in both groups had paroxysmal AF (56\% vs $48 \%, P=0.63)$.

\section{Echocardiography}

The results of the echocardiographic measurements are shown in Table 2 . The ratio of early diastolic mitral flow velocity and mitral annular early diastolic velocity (E/e' ratio) was significantly lower in group 1 than in group 2 (6.9 \pm 1.4 vs $8.3 \pm 2.2, P=0.034)$. Left ventricular end systolic volumes were higher in group $1(49 \pm 21 \mathrm{ml} v s 38 \pm 14 \mathrm{ml}, P$ $=0.048$ ). There was no difference between the groups in LA diameter, LVEF, LV dimensions, LV diastolic volume or mass index, transmitral flow propagation velocities and PV flow indexes.

Multivariate analysis was performed for variables with a $\mathrm{P}$ value of < 0.1 (i.e., female gender, CV history, LV end diastolic and systolic volumes, E wave deceleration time and E/e'). In the multivariate model, the E/e' ratio was the most powerful predictor of AF symptom recurrence (OR 1.911; 95\% CI 1.046-3.493, $\mathrm{P}=0.035$ ) (Table 3). The area under the receiver operating characteristic (ROC) curve was 0.68 (95\% CI 0.51-0.84) indicating that the E/e'ratio might 
predict the outcome of AF ablation, but its accuracy in this relative small population was not good.

Table 1. Baseline Characteristics

\begin{tabular}{|l|c|c|c|c|}
\hline & $\begin{array}{c}\text { Total } \\
(\mathbf{n = 4 9})\end{array}$ & $\begin{array}{c}\text { Group 1 } \\
(\mathbf{n = 1 8})\end{array}$ & $\begin{array}{c}\text { Group 2 } \\
(\mathbf{n = 3 1})\end{array}$ & P Value \\
\hline \hline Age & $53 \pm 8$ & $52 \pm 8$ & $54 \pm 8$ & 0.47 \\
\hline Female & $7(14 \%)$ & $0(0 \%)$ & $7(23 \%)$ & 0.038 \\
\hline HTA & $10(20 \%)$ & $3(17 \%)$ & $7(23 \%)$ & 0.73 \\
\hline CAD & $3(6 \%)$ & $1(6 \%)$ & $2(7 \%)$ & 1.00 \\
\hline Lung disease & $3(6 \%)$ & $0(0 \%)$ & $3(10 \%)$ & 0.29 \\
\hline Systolic BP & $134 \pm 16$ & $132 \pm 15$ & $136 \pm 17$ & 0.39 \\
\hline Diastolic BP & $81 \pm 12$ & $78 \pm 12$ & $82 \pm 11$ & 0.29 \\
\hline BMI & $27 \pm 4$ & $26 \pm 4$ & $28 \pm 4$ & 0.31 \\
\hline AADs prior ablation & $1.6 \pm 1.0$ & $1.5 \pm 0.8$ & $1.6 \pm 1.1$ & 0.82 \\
\hline AADs at procedure & $23(47 \%)$ & $11(61 \%)$ & $12(39 \%)$ & 0.13 \\
\hline $\begin{array}{l}\text { Flecainide } \\
\text { Amiodarone }\end{array}$ & $10(20 \%)$ & $2(11 \%)$ & $8(26 \%)$ & 0.22 \\
\hline Antihypertensive drugs & $1.1 \pm 0.7$ & $1.1 \pm 0.8$ & $1.1 \pm 0.7$ & 0.93 \\
\hline History of AF (yrs.) & $7.3 \pm 5.5$ & $6.0 \pm 4.7$ & $8.0 \pm 5.9$ & 0.22 \\
\hline Paroxysmal AF & $25(51 \%)$ & $10(56 \%)$ & $15(48 \%)$ & 0.63 \\
\hline Persistent AF & $17(35 \%)$ & $5(28 \%)$ & $12(39 \%)$ & 0.54 \\
\hline Long-standing persistent AF & $7(14 \%)$ & $3(17 \%)$ & $4(13 \%)$ & 0.72 \\
\hline History of CV & $30(61 \%)$ & $7(39 \%)$ & $22(73 \%)$ & 0.032 \\
\hline AF at procedure & $16(33 \%)$ & $6(46 \%)$ & $10(32 \%)$ & 0.50 \\
\hline Procedure duration (min) & $177 \pm 30$ & $175 \pm 34$ & $179 \pm 27$ & 0.78 \\
\hline RF time (min) & $52 \pm 11$ & $53 \pm 10$ & $51 \pm 13$ & 0.68 \\
\hline
\end{tabular}

HTA, hypertension; CAD, coronary artery disease; BP, blood pressure; BMI, body mass index; $\mathrm{AAD}$, antiarrhythmic drug; $\mathrm{AF}$, atrial fibrillation; $\mathrm{CV}$, cardioversion; $\mathrm{RF}$, radiofrequency.

\section{DISCUSSION}

In our study, the left ventricular filling pressure measured by the E/e' ratio was an independent and most powerful predictor for $\mathrm{AF}$ reoccurrence following single ablation procedure at one year. In addition, transmitral $\mathrm{E}$ wave deceleration time was longer in patients with AF relapse, but the traditional risk factors, such as LA size, had no predictive value. However, patients with large LA diameter (> $50 \mathrm{~mm})$ and chronic heart failure were excluded.

In addition to the classical risk factors for AF relapse, other underlying conditions [14, 15], imaging findings [12, 16-18], biomarkers [19, 20] and genetic factors [21] have been noted to predict AF reoccurrence after catheter ablation. Still, the question remains, how we can better identify patients with increased risk for AF relapses. The level of atrial fibrosis has been shown to correlate with AF ablation outcome. Detection of the extent of fibrosis in the atria is possible with a specific delayed enhancement MRI technique [12]. As a non-invasive and more readily available diagnostic tool, echocardiography might provide a more practical preoperative means to predict AF ablation outcome.
In this study, the E/e' ratio was an independent predictor for long-term success of AF ablation. Unlike many other diastolic measurements, which are affected by AF, the E/e' ratio can be measured also during AF [22].

Table 2. Echocardiographic Data

\begin{tabular}{|l|c|c|c|c|}
\hline & $\begin{array}{c}\text { Total } \\
(\mathbf{n = 4 9})\end{array}$ & $\begin{array}{c}\text { Group 1 } \\
(\mathbf{n = 1 8})\end{array}$ & $\begin{array}{c}\text { Group 2 } \\
(\mathbf{n = 3 1})\end{array}$ & P Value \\
\hline \hline LVEF $(\%)$ & $62 \pm 8$ & $62 \pm 8$ & $62 \pm 7$ & 0.97 \\
\hline LVEDD $(\mathrm{mm})$ & $50 \pm 8$ & $50 \pm 5$ & $50 \pm 10$ & 0.95 \\
\hline LVEDV $(\mathrm{ml})$ & $108 \pm 37$ & $123 \pm 47$ & $99 \pm 28$ & 0.076 \\
\hline LVESV $(\mathrm{ml})$ & $42 \pm 17$ & $49 \pm 21$ & $38 \pm 14$ & 0.048 \\
\hline LVMI & $116 \pm 33$ & $124 \pm 32$ & $112 \pm 34$ & 0.24 \\
\hline LA size $(\mathrm{mm})$ & $41 \pm 5$ & $41 \pm 5$ & $41 \pm 5$ & 0.97 \\
\hline RVDD $(\mathrm{mm})$ & $27 \pm 5$ & $29 \pm 6$ & $26 \pm 5$ & 0.12 \\
\hline E velocity $(\mathrm{cm} / \mathrm{s})$ & $71 \pm 15$ & $68 \pm 9$ & $73 \pm 17$ & 0.19 \\
\hline E Deceleration time $(\mathrm{ms})$ & $149 \pm 32$ & $138 \pm 29$ & $157 \pm 31$ & 0.057 \\
\hline E/A velocity ratio & $1.4 \pm 0.5$ & $1.4 \pm 0.4$ & $1.4 \pm 0.5$ & 0.85 \\
\hline e' (cm/s) & $9.4 \pm 2.2$ & $10 \pm 1.8$ & $9.0 \pm 2.3$ & 0.18 \\
\hline E/e' & $7.8 \pm 2.1$ & $6.9 \pm 1.4$ & $8.3 \pm 2.2$ & 0.034 \\
\hline Vp (cm/s) & $58 \pm 21$ & $51 \pm 14$ & $61 \pm 23$ & 0.13 \\
\hline IVRT (ms) & $90 \pm 15$ & $90 \pm 15$ & $90 \pm 15$ & 0.98 \\
\hline S velocity (cm/s) & $53 \pm 14$ & $53 \pm 11$ & $52 \pm 16$ & 0.82 \\
\hline D velocity (cm/s) & $54 \pm 14$ & $51 \pm 14$ & $56 \pm 14$ & 0.31 \\
\hline Ar velocity (cm/s) & $28 \pm 8$ & $26 \pm 6$ & $29 \pm 9$ & 0.41 \\
\hline
\end{tabular}

LVEF, left ventricular ejection fraction; LVEDD, left ventricular end diastolic diameter; LVEDV, left ventricular end diastolic volume; LVESV, left ventricular end systolic volume; LVMI, left ventricular mass index; LA, left atrial; RVDD; right ventricular diastolic diameter; E, early diastolic mitral flow velocity; A, late diastolic mitral flow velocity; e', mitral annular early diastolic velocity; Vp, transmitral flow propagation velocity; IVRT, isovolumic relaxation time; S, systolic pulmonary vein flow; D, early diastolic pulmonary venous flow; Ar, pulmonary venous atrial reversal flow.

Table 3. Independent Predictors of AF Recurrence in Multivariate Analysis

\begin{tabular}{|c|c|c|c|}
\hline & OR & $\mathbf{9 5 \%}$ CI & P Value \\
\hline \hline E/e' & 1.911 & $1.046-3.493$ & 0.035 \\
\hline E Deceleration time & 1.028 & $1.000-1.056$ & 0.046 \\
\hline
\end{tabular}

In keeping with our data, results of some previous studies indicate that diastolic dysfunction or heart failure predicts AF recurrence after ablation [23-26]. It has been shown that LV diastolic dysfunction may promote AF [27]. On the other hand, it is known that the atria are more susceptible to subtle diastolic changes than the ventricles [28]. Nagueh, et al. [29] showed that the E/e' ratio was linearly correlated with LA pressure. In a recent study, invasively measured LA pressure was significantly higher in patients with persistent $v s$ paroxysmal AF [30]. It remains unclear whether the elevation of the E/e' ratio is due to diastolic dysfunction caused by subclinical underlying conditions, genetic factors 
or higher preoperative AF burden in patients with no response to $\mathrm{AF}$ ablation. In keeping with the last hypothesis, we have shown earlier that patients with frequent episodes of symptomatic AF had reduced systolic PV flow during normal sinus rhythm compared to those with few or no episodes of paroxysmal AF [31]. It would be interesting to evaluate whether there is a correlation between the E/e 'ratio and the extent of fibrosis detected by late gadolinium enhancement MRI.

\section{STUDY LIMITATIONS}

The study population was rather small and did not allow separate comparison of patients with paroxysmal $v s$ persistent or long-standing persistent AF. Moreover, patients had minor comorbidity. Therefore, one needs to be cautious when extrapolating these results to other patient populations. Nevertheless, our data indicate that in selected cases, simple preoperative echocardiographic measurements may be valuable in tailoring the treatment of the patients. Furthermore, because control electrophysiological study was not performed, the mechanisms behind the AF relapses could not be specified. It is possible that the patients with elevated E/e'ratio have higher risk for PV reconnection or that they require more extensive substrate modification like those with long-standing persistent AF. Finally, despite the apparent clinical benefit, it should be noted that asymptomatic AF episodes may have been under-detected and true AF burden could not be estimated since no continuous ECG monitoring with implantable device was used.

\section{CONCLUSIONS}

Our data indicate that E/e' ratio is a powerful predictor of AF reoccurrence after PVI. As a non-invasive, readily available and inexpensive measurement, E/e'ratio is a practical tool to recognize the patients in risk for treatment failure and to refine treatment strategy. Further studies are needed to evaluate its relation to atrial fibrosis and $\mathrm{AF}$ burden and to define its role in guiding the treatment strategy.

\section{CONFLICT OF INTEREST}

M.J.P.R. has been consulting in clinical cases where the Carto $^{\mathrm{TM}}$ system might be useful. The other author (s) declare that they have no competing interests.

\section{ACKNOWLEDGEMENTS}

This study was supported by Finnish Foundation for Cardiovascular Research and Instrumentarium Science Foundation.

\section{REFERENCES}

[1] Camm AJ, Lip GY, De Caterina R, et al. 2012 focused update of the ESC Guidelines for the management of atrial fibrillation: an update of the 2010 ESC Guidelines for the management of atrial fibrillation. Developed with the special contribution of the European Heart Rhythm Association. Eur Heart J 2012; 33 (21): 2719-47.

[2] Stewart S, Hart CL, Hole DJ, McMurray JJ. A population-based study of the long-term risks associated with atrial fibrillation: 20year follow-up of the Renfrew/Paisley study. Am J Med 2002; 113 (5): 359-64.

[3] Benjamin EJ, Wolf PA, D'Agostino RB, Silbershatz H, Kannel WB, Levy D. Impact of atrial fibrillation on the risk of death: the Framingham Heart Study. Circulation 1998; 98 (10): 946-52.
[10] Winkle RA, Mead RH, Engel G, Patrawala RA. Long-term results of atrial fibrillation ablation: the importance of all initial ablation failures undergoing a repeat ablation. Am Heart J 2011; 162 (1): 193-200

[11] Ouyang F, Tilz R, Chun J, et al. Long-term results of catheter ablation in paroxysmal atrial fibrillation: lessons from a 5-year follow-up. Circulation 2010; 122 (23): 2368-77.

[12] Mahnkopf C, Badger TJ, Burgon NS, et al. Evaluation of the left atrial substrate in patients with lone atrial fibrillation using delayed-enhanced MRI: implications for disease progression and response to catheter ablation. Heart Rhythm 2010; 7 (10): 1475-81.

[13] Hakalahti A, Uusimaa P, Ylitalo K, Raatikainen MJP. Catheter ablation of atrial fibrillation in patients with therapeutic oral anticoagulation treatment. Europace 2011; 13 (5): 640-5.

[14] Chang SL, Tuan TC, Tai CT, et al. Comparison of outcome in catheter ablation of atrial fibrillation in patients with $v s$ without the metabolic syndrome. Am J Cardiol 2009; 103 (1): 67-72.

[15] Jongnarangsin K, Chugh A, Good E, et al. Body mass index, obstructive sleep apnea, and outcomes of catheter ablation of atrial fibrillation. J Cardiovasc Electrophysiol 2008; 19 (7): 668-72.

[16] Helms AS, West JJ, Patel A, et al. Relation of left atrial volume from three-dimensional computed tomography to atrial fibrillation recurrence following ablation. Am J Cardiol 2009; 103 (7): 989-93.

[17] Oakes RS, Badger TJ, Kholmovski EG, et al. Detection and quantification of left atrial structural remodeling with delayedenhancement magnetic resonance imaging in patients with atrial fibrillation. Circulation 2009; 119 (13): 1758-67.

[18] Wong CX, Abed HS, Molaee P, et al. Pericardial fat is associated with atrial fibrillation severity and ablation outcome. J Am Coll Cardiol 2011; 57 (17): 1745-51.

[19] Hussein AA, Saliba WI, Martin DO, et al. Plasma B-type natriuretic peptide levels and recurrent arrhythmia after successful ablation of lone atrial fibrillation. Circulation 2011; 123 (19): 207782.

[20] Lin YJ, Tsao HM, Chang SL, et al. Prognostic implications of the high-sensitive C-reactive protein in the catheter ablation of atrial fibrillation. Am J Cardiol 2010; 105 (4): 495-501.

[21] Husser D, Adams V, Piorkowski C, Hindricks G, Bollmann A. Chromosome $4 \mathrm{q} 25$ variants and atrial fibrillation recurrence after catheter ablation. J Am Coll Cardiol 2010; 55 (8): 747-53.

[22] Al-Omari MA, Finstuen J, Appleton CP, Barnes ME, Tsang TS Echocardiographic assessment of left ventricular diastolic function and filling pressure in atrial fibrillation. Am J Cardiol 2008; 101 (12): 1759-65.

[23] Li C, Ding X, Zhang J, Zhou C, Chen Y, Rao L. Does the E/e' index predict the maintenance of sinus rhythm after catheter ablation of atrial fibrillation? Echocardiography 2010; 27 (6): 6306.

[24] Hu YF, Hsu TL, Yu WC, et al. The impact of diastolic dysfunction on the atrial substrate properties and outcome of catheter ablation in patients with paroxysmal atrial fibrillation. Circ J 2010; 74 (10): 2074-8. 
[25] Cha YM, Wokhlu A, Asirvatham SJ, et al. Success of ablation for atrial fibrillation in isolated left ventricular diastolic dysfunction: a comparison to systolic dysfunction and normal ventricular function. Circ Arrhythm Electrophysiol 2011; 4 (5): 724-32.

[26] Ejima K, Shoda M, Arai K, et al. Impact of diastolic dysfunction on the outcome of catheter ablation in patients with atrial fibrillation. Int J Cardiol 2013; 164 (1): 88-93.

[27] Tsang TS, Gersh BJ, Appleton CP, et al. Left ventricular diastolic dysfunction as a predictor of the first diagnosed nonvalvular atrial fibrillation in 840 elderly men and women. J Am Coll Cardiol 2002; 40 (9): 1636-44.

[28] Nattel S, Burstein B, Dobrev D. Atrial remodeling and atrial fibrillation: mechanisms and implications. Circ Arrhythm Electrophysiol 2008; 1 (1): 62-73.
[29] Nagueh SF, Mikati I, Kopelen HA, Middleton KJ, Quinones MA, Zoghbi WA. Doppler estimation of left ventricular filling pressure in sinus tachycardia. A new application of tissue doppler imaging. Circulation 1998; 98 (16): 1644-50.

[30] Yoshida K, Ulfarsson $\mathrm{M}$, Oral $\mathrm{H}$, et al. Left atrial pressure and dominant frequency of atrial fibrillation in humans. Heart Rhythm 2011; 8 (2): 181-7.

[31] Lindgren KS, Pekka Raatikainen MJ, Juhani Airaksinen KE, Huikuri HV. Relationship between the frequency of paroxysmal episodes of atrial fibrillation and pulmonary venous flow pattern. Europace 2003; 5 (1): 17-23.

(c) Hakalahti et al.; Licensee Bentham Open.

This is an open access article licensed under the terms of the Creative Commons Attribution Non-Commercial License (http://creativecommons.org/licenses/by-nc/3.0/) which permits unrestricted, non-commercial use, distribution and reproduction in any medium, provided the work is properly cited. 